\title{
Objetivos Educacionais na Pedagogia das Humanidades Médicas: Taxonomias Alternativas (Campos de Significado e Competências)
}

\author{
Educational Objectives in the Teaching of \\ Medical Humanities: Alternative Taxonomies \\ (Realms of Meanings and Competencies)
}

Ricardo Tapajós ${ }^{I}$

\footnotetext{
PALAVRAS-CHAVE:

- Humanidades/tendências;

- Humanidades/educação;

- Currículo/tendências.
}

KEY WORDS:

- Humanities/trends;

- Humanities/education;

- Curriculum/trends.

Recebido em: 07/04/2007

Aprovado em: 21/06/2007

\section{RESUMO}

Os currículos médicos estão preocupados em formar médicos detentores de traços humanísticos, que passam, assim, a ser objetivos educacionais. Deve-se desenvolver, então, a sua pedagogia. Como objetivos, podem ser tratados pedagogicamente com base nos corpos conceituais teóricos que informam o planejamento de currículos, como a taxonomia de objetivos educacionais clássica, que compreende os domínios cognitivo, afetivo e psicomotor. Esta taxonomia dá conta de muitos dos objetivos relacionados aos traços humanísticos, organizando-os e facilitando a tarefa de planejamento educacional nessa área, mas deixa marginalizados alguns aspectos do conhecimento humano cruciais para as Humanidades, como os objetivos que se referem a autoconhecimento, amadurecimento e à individuação, reconhecimento dos próprios sentimentos e habilidades de comunicação interpessoal. Devem-se, então, buscar outros sistemas conceituais como referenciais teóricos mais adequados às Humanidades. É possível que se possa usar as taxonomias de maneira aditiva, procurando objetivos humanísticos em cada uma das categorias e subcategorias das diversas taxonomias. Assim, aumenta-se a abrangência do corpo de objetivos educacionais de natureza humanística.

\begin{abstract}
Medical curricula are concerned with preparing physicians with humanistic traits. Thus, these traits become educational objectives, for which a teaching method needs to be developed. As objectives, they can be handled pedagogically on the basis of the theoretical conceptual body that informs curriculum planning such as the classical taxonomy of educational objectives. This taxonomy can cover a great number of objectives related to these humanistic traits, organizing them and making the task of curriculum planning in this area easier; it leaves unmapped however some crucial aspects of human knowledge, such as objectives regarding self-knowledge, self-development, individuation, awareness of personal feelings and inter-personal communication skills. Thus, other conceptual systems with theoretical references more adequate for the Humanities. Should be considered. Both are reviewed in this paper. These conceptual systems should be used in addition to each other and to the classical taxonomy, allowing humanistic objectives to be sought in each of their categories and subcategories. This way, the range of humanistic teaching objectives can be increased.
\end{abstract}




\section{INTRODUÇÃO}

O produto da educação de profissionais de saúde deve ser um profissional dotado de traços humanísticos. Se a pedagogia dessa educação repousa (tylerianamente) sobre a enunciação de objetivos educacionais, é lícito entender que os objetivos educacionais das Humanidades médicas se devam referir justamente a esses traços humanísticos desejados e a sua aquisição ou desenvolvimento. Rotineiramente, esses objetivos são elencados sob um referencial teórico clássico, a taxonomia de objetivos educacionais, e divididos em três domínios (cognitivo, afetivo e psicomotor).

Entretanto, é extensa a lista dos traços humanísticos que o médico deve exibir para ser considerado um bom profissional. Muitos desses traços podem, de fato, ser contemplados pela taxonomia de objetivos educacionais em algum de seus domínios. Por exemplo, os traços relacionados aos processos do pensar, que envolvem pensamento mais crítico e reflexivo, são enunciáveis como objetivos educacionais no domínio cognitivo. Os traços que envolvem valores e atitudes e sua percepção nos outros seres e nas sociedades podem ser expressos como objetivos no domínio afetivo.

Isto confirma a utilidade desse sistema taxonômico para o planejamento educacional na área das Humanidades médicas. Isto não constitui uma inovação, na medida em que o uso da taxonomia de objetivos educacionais é clássico em Educação e praxe em educação médica.

Entretanto, o uso dessa taxonomia, apesar de costumeiro, deixa marginalizados alguns aspectos do conhecimento humano cruciais para as Humanidades. De fato, alguns dos traços humanísticos necessários ao bom profissional têm inserção difícil no esqueleto dessa taxonomia. Onde exatamente colocar os objetivos que se referem ao autoconhecimento, ao amadurecimento e à individuação, ao reconhecimento dos próprios sentimentos e às habilidades de comunicação interpessoal?

Passa a ser, portanto, desejável, procurar e utilizar outras sistemáticas taxonômicas que dêem conta, total ou parcialmente, da diversidade de objetivos educacionais que passaram a ser necessários, no modelo humanista, para se formar um bom profissional. Essas taxonomias existem e algumas já foram compiladas no contexto da educação médica ${ }^{1,2}$. Entretanto, é no contexto da educação de enfermagem que elas aparecem aplicadas de maneira mais sistematizada, especificamente no caso dos Campos de Significado de Phenix ${ }^{3,4,5}$ e das Competências ${ }^{6}$.

Ainda que relativamente estranho ao planejamento dos currículos médicos, o uso dessas taxonomias alternativas, integradas à prática educacional, pode constituir um primeiro passo criativo para o "projeto pedagógico genuíno e integra- do $^{17}$ que se fez necessário para resolver a questão da formação de bons profissionais, isto é, médicos humanizados.

Assim, levantaram-se, na literatura da educação de profissionais da saúde, objetivos que pudessem ser expressos em termos das categorias dessas taxonomias alternativas. Com isso, procura-se mostrar que o uso de outras taxonomias pode ampliar o arcabouço organizacional de objetivos necessários, numa concepção tyleriana de educação, para dar conta dos traços humanísticos desejados no produto final da educação médica.

\section{A TAXONOMIA DE PHENIX: CAMPOS DE SIGNIFICADO}

Os Campos de Significado (Realms of Meaning) ${ }^{8}$ são um modo menos prevalente de sistematizar objetivos. Ainda assim, eles têm-se aplicado em currículos escolares gerais ${ }^{9}$, currículos de educação médica ${ }^{1}$ e de educação de enfermagem ${ }^{3}$, sempre correlacionando-se, desde a sua criação, a uma formação generalista.

Phenix $(1961)^{10}$ baseia seus conceitos de Educação nos valores humanos e em sua transformação. Educação é uma “empreitada moral"10 - o termo "moral" referindo-se a uma "conduta proposital baseada na consideração de valores" - cuja meta é transformar as pessoas em direção a um bem comum e cujo racional prevê valores merecedores de "devoção"10. Recomenda que as escolhas curriculares sejam baseadas nas exigências impostas pelo desenvolvimento da civilização moderna e que o reconhecimento de valores seja um princípio curricular. Esse posicionamento educacional é bastante compatível, senão mesmo desejável, com as Humanidades médicas, e o uso desse sistema taxonômico deve ser incentivado, por suas potencialidades.

Tendo desenvolvido um sistema coerente de idéias por meio do qual todas as partes diferentes de um currículo podem ser identificadas ${ }^{9}$, Phenix $(1964)^{8}$ propôs seis áreas básicas de conhecimento e significado, derivadas da experiência humana, sob as quais os objetivos podem ser alocados. Chamou essas áreas de Campos de Significado, que são os seguintes:

- Campo Simbólico (Symbolics): ocupa-se em expressar e comunicar significado. Compreende as linguagens, inclusive a matemática e a comunicação não discursiva (linguagem corporal, costumes e ritos);

- Campo Empírico (Empirics): envolve a lógica da ciência e da generalização ${ }^{3}$, utilizando descrição, observação e experimentação. As Ciências Físicas e Naturais podem ser incluídas aqui, bem como as Ciências Humanas e Sociais;

- Campo Estético (Aesthetics): envolve a significação não generalizável, mas singular. Cada experiência pode 
ser única e incomparável, como no caso das artes de maneira geral;

- Campo Sinoético (Synnoetics): é associado ao conhecimento pessoal e tácito de si mesmo, dos outros e das coisas. Envolve fatos singulares, insight, introspecção e conscientização. O conhecimento nesse campo não é imparcial e objetivo, mas subjetivo e comprometido;

- Campo Ético (Ethics): envolve conhecimento moral e conduta pessoal baseada em decisões livres, responsáveis e deliberadas;

- Campo Sinóptico (Synoptics): compreende significados inclusivos e integrados e combina conhecimentos empíricos, estéticos e sinoéticos para dar conta da experiência humana. Abrange a História, a Religião e a Filosofia.

Não há uma hierarquia óbvia nesses campos. Entretanto, uma seqüência ordenada parece existir do Campo Simbólico ao Sinóptico ${ }^{3,9}$. Um currículo que visa a uma educação generalista deve ofertar objetivos em todos os Campos'. A inclusão de cada um dos Campos nos currículos de profissionais de saúde facilita a inclusão de todo um espectro de educação generalista $^{3}$. No campo da educação de enfermagem, esse conceito é duplamente citado na literatura: o profissional de enfermagem competente deverá exibir habilidades e conhecimentos em quatro campos de conhecimento: empírico (refletindo a ciência da enfermagem), estético (refletindo a arte da enfermagem), pessoal (refletindo o autoconhecimento) e moral (englobando conhecimentos de natureza ética) ${ }^{4,5}$.

Objetivos educacionais compilados na literatura e pertinentes aos Campos de Significado de Phenix são citados a seguir, como ilustração. Tradicionalmente, os objetivos no Campo Ético têm representação abundante nos currículos. Por sua extensão e prevalência, fogem do escopo deste levantamento. Por ora, basta sumarizar que lidam com decisões informadas, esclarecidas e livres, enfim, com questões morais ${ }^{11,12}$, inclusas a pesquisa biomédica e a relação médico-paciente ${ }^{13}$.

Em termos do Campo Estético, os alunos poderão, com a ajuda das Humanidades, lidar com significados singulares, particulares, não científicos e subjetivos do fenômeno humano; exercer atividades contemplativas ${ }^{14}$; encontrar representações estéticas e simbólicas ${ }^{15}$; iniciar-se nos domínios artístico e estético sem querer ser artista ou poeta ${ }^{16}$.

É no Campo Sinoético que se concentram muitos objetivos educacionais. Com a ajuda das Humanidades, os alunos poderão fomentar autoconhecimento e auto-entendimento ${ }^{13,16-}$ 21; auto-escrutínio ${ }^{22}$ e introspecção ${ }^{23,24}$. Poderão direcionar sua atenção não só ao paciente, mas também a si $\operatorname{mesmos}^{17}$. Po- derão tentar manter-se em "escala" ou "medida" adequada, evitando pretensão, arrogância, ganância e desmesura ${ }^{25}$.

Em termos de crescimento pessoal, individuação, satisfação e bem-estar pessoal, com a ajuda das Humanidades os alunos deverão ser capazes de fomentar crescimento pessoal ${ }^{26}$; usar oportunidades de amadurecer ${ }^{27}$; ter mais background para a resolução de conflitos pessoais ${ }^{16}$; tentar ser alguém bom ${ }^{28}$; viver vidas enriquecidas e mais plenas ${ }^{11,29-32}$; perceber o valor da própria vida ${ }^{33}$; desenvolver uma atitude saudável de mente $^{14}$; restaurar um senso de equilíbrio ${ }^{34}$; separar seus valores e experiências daqueles dos outros ${ }^{35}$; promover individualida$\mathrm{de}^{16}$; entender o próprio processo de transição de aluno para profissional $^{19}$ e examinar e aprender com as próprias reações ao trabalho, aos colegas, aos pacientes e suas famílias ${ }^{16}$.

Ainda no Campo Sinoético estão os objetivos relacionados à vocação e à construção de uma carreira plena e satisfatória. Os alunos deverão ser capazes de derivar gratificação pessoal da profissão ${ }^{25}$; preparar-se para a própria carreira ${ }^{36}$; entender as diferenças entre uma carreira e uma sucessão de empregos, uma carreira e uma fonte geradora de provimentos e uma carreira e profissionalismo ${ }^{37}$; discutir sucesso em termos não só de sucesso pessoal, mas também da extensão do cumprimento de metas profissionais ${ }^{37}$; fazer escolhas de carreira ${ }^{37}$ e especialização, e aumentar a qualidade de vida ${ }^{34}$.

\section{A TAXONOMIA DE MORSE ET AL. (1992): COMPETÊNCIAS}

Uma forma de conceber objetivos em programas educacionais é delinear os resultados esperados da preparação profissional em termos de competências ${ }^{6}$. Esse arcabouço teórico deriva de um modelo conceitual desenvolvido para o estudo da preparação profissional. Há duas categorias de resultados profissionais esperados: competências profissionais específicas e competências adicionais. As primeiras lidam com o entendimento conceitual e padrões de performance técnica necessários à profissão, enquanto as últimas se referem a outras habilidades igualmente necessárias para atingir a competência técnico-profissional.

Essas competências podem ser subdivididas ainda mais, para oferecer um arcabouço para a identificação, classificação e análise de produtos educacionais desejados ${ }^{6}$.

\section{Competências profissionais específicas}

- Competência conceitual: entendimento das fundações teóricas da profissão;

- Competência técnica: em termos da execução das tarefas profissionais requeridas;

- Competência integrativa: amalgamamento entre teoria e habilidades técnicas na prática real; 
- Disponibilidade para o mercado (career marketability): tornar-se disponível para o mercado (marketable), como resultado da educação e treinamento adquiridos.

\section{Competências adicionais}

- Competência comunicacional: uso efetivo da comunicação escrita e verbal;

- Pensamento crítico: exame racional, lógico e coerente dos assuntos;

- Competência contextual: reconhecimento do contexto ambiental e social em que a profissão é praticada;

- Sensibilidade estética: consciência das artes e do comportamento humano para fins de enriquecimento pessoal e aplicação na melhoria da profissão;

- Identidade profissional: preocupação com a melhoria do conhecimento, habilidades e valores da profissão;

- Ética profissional: entendimento e aceitação da ética da profissão como padrão que guarda o comportamento profissional;

- Competência adaptativa: habilidade de antecipar, adaptar-se a e/ou promover mudanças importantes para a profissão;

- Capacidade de liderança: contribuição como membro produtivo da profissão, além da capacidade de assumir papéis de liderança apropriados à profissão e à sociedade;

- Preocupação acadêmica com melhoria: reconhecimento da necessidade de produzir conhecimento na profissão por meio de pesquisa;

- Motivação para aprendizagem continuada (lifelong learning competence): desejo de continuar atualizando conhecimentos e habilidades.

Esse arcabouço pode ser útil para guiar a avaliação de resultados educacionais, planejamento de currículo, pesquisa em educação de profissões de saúde e facilitação de conexões curriculares dentro das profissões e entre elas ${ }^{6}$.

Objetivos nos campos da competência conceitual, competência técnica e pensamento crítico são geralmente mapeados nos domínios cognitivo e psicomotor. Objetivos na competência integrativa tendem a ser mais interessantes, já que prevêem a transposição da teoria à prática, ou a aplicação dos conceitos e pensamentos teóricos (entenda-se científicos) da Medicina à prática médica, ou ao fenômeno humano singular que é o objeto desta prática. As Humanidades ajudariam justamente na integração entre a "ciência" e a "arte" do cuidado de saúde $12,15,16,34,38$.

Objetivos no campo da disponibilidade para o mercado (career marketability) assumem o formato: "no final do curso, o aluno deverá estar de posse deste ou daquele traço necessário para estar mais disponível para o mercado (marketability)". O próprio pressuposto com que se trabalhou nesse capítulo é de que o profissional desejado pelo mercado, após a mudança para o paradigma humanista, seja detentor de traços técnicos de excelência, mas também de traços humanísticos. Esses traços são aqueles que as Humanidades ensejam.

Com a ajuda das Humanidades, os alunos poderão cumprir objetivos importantíssimos no campo da competência comunicacional. Poderão exercitar sua capacidade de comunicar ${ }^{15}$; de entender os desejos pobremente verbalizados de pacientes ${ }^{30}$ e de se comunicar socialmente com outros ${ }^{16,39}$. Poderão desenvolver sensibilidades a "signos obscuros, oblíquos ou não verificáveis" ${ }^{\prime 40}$ e estabelecer comunicação ótima entre profissional e paciente ${ }^{41}$.

Ainda com a ajuda das Humanidades, os alunos poderão cumprir objetivos no campo da competência contextual, lidando com insights na sociedade e seu funcionamento em termos gerais e em termos da profissão médica. Poderão exercitar formas de lidar com problemas sociais gerais ${ }^{11,29}$; ganhar conhecimento da sociedade ${ }^{11}$; examinar o lugar do indivíduo na sociedade ${ }^{16}$; mostrar uma atitude reflexiva sobre assuntos sociais cruciais ${ }^{15}$; entender o sentido de mudança na sociedade presente e futura ${ }^{41}$; examinar como as idéias e ideais da nossa civilização são usados como base para o entendimento de outras culturas ${ }^{16}$; aumentar a própria consciência social ${ }^{16}$; delinear os valores compartilhados que unem as pessoas enquanto sociedade $^{16}$; examinar o contexto social das doenças, da cura ${ }^{13}$ e do cuidado de saúde ${ }^{42}$; locar os eventos da Medicina num contexto cultural e humano mais amplo ${ }^{43}$; e estar ciente da universidade e seus problemas ${ }^{14}$.

No campo da identidade profissional, os alunos poderão, com a ajuda das Humanidades, examinar seu próprio papel social enquanto profissionais e examinar o papel social do profissional de saúde em geral.

Em termos do próprio papel social enquanto médicos, poderão medir quem são e o peso do que fazem na sociedade de que participam ${ }^{25}$; entender o contexto cultural do profissional de saúde ${ }^{19}$; entender os deveres de um profissional de saúde ${ }^{15}$; delinear o seu lugar dentro da profissão ${ }^{19,37}$; ver as próprias atividades nos contextos humano, físico e social ${ }^{44}$; preparar-se para cidadania futura e efetiva na profissão e na sociedade ${ }^{37}$; desenvolver novos papéis do profissional de saúde no diagnóstico, tratamento e atribuições sanitárias ${ }^{41}$. Podem ainda examinar a própria contribuição à sobrevivência e viabilidade da profissão ${ }^{37}$, tomando atitudes e decisões norteadas mais pelo bem-estar da profissão do que por interesses próprios $^{37}$. 
Em relação ao exame do papel social do profissional de saúde, deverão, com o auxílio das Humanidades, avaliar a profissão e suas responsabilidades sociais ${ }^{18,19}$; entender o construto social dentro do qual a profissão de saúde existe ${ }^{19}$; ter imagens do que é o "bom profissional" e quais as idéias que adequadamente estruturam a profissão ${ }^{20}$; adquirir uma percepção mais profunda da própria natureza da Medicina, suas pressuposições filosóficas, modos de raciocínio, bases epistêmicas e desenvolvimento histórico e intelectual ${ }^{11}$; inquirir os usos da Medicina para os indivíduos e a sociedade ${ }^{11}$; examinar a profissão $\mathrm{o}^{20}$; apreciar a profissão no seu contexto social mais amplo ${ }^{6}$; examinar o desenvolvimento da profissão médica e seu relacionamento com a sociedade ${ }^{13}$.

No campo da motivação para aprendizagem continuada (lifelong learning), com a ajuda das Humanidades os alunos poderão construir um arcabouço para a aquisição contínua de conhecimentos sobre si mesmos e sobre o mundo ${ }^{16,45}$.

No campo da competência adaptativa, com o auxílio das Humanidades, os alunos poderão desenvolver capacidades adaptativas de maneira geral ${ }^{27}$, bem como adaptar-se a uma sociedade e a uma profissão que estão sofrendo mudanças rápidas $^{35}$.

\section{O DOMÍNIO EMOCIONAL DE DAVIES}

O domínio afetivo ${ }^{46}$ não lida com afetos, mas com valores e atitudes. Quando se julga importante lidar educacionalmente com afetos, pode-se recorrer ao domínio emocional ${ }^{9}$, que compila, de maneira frouxamente hierárquica e pouco aglutinada, sentimentos de prazer, desprazer e realização. $\mathrm{O}$ importante nesse domínio é nomear sentimentos e reconhecê-los em si ou em outrem, muito mais que classificá-los. Citam-se estímulo, excitação, aflição, encanto, medo, desgosto, raiva, afeição, entusiasmo, ciúmes, amor, contentamento, júbilo, satisfação e êxtase .

Enunciar objetivos educacionais no domínio emocional parece necessário, uma vez que as escolas médicas ocidentais freqüentemente "destroem o médico" que queira ser humanizado, juntamente com "qualquer senso natural de sentimento" ${ }^{47}$, o que resulta em ansiedade ${ }^{48}$ e num efeito emocional desumanizador $^{49,50}$ para o aluno em longo período de treinamento.

Em termos da expressão, reconhecimento e nomeação de sentimentos, os alunos poderão, com a ajuda das Humanidades, aumentar a percepção de sentimentos; reconhecer a importância dos sentimentos na vida profissional; contrapor-se à tendência de suprimir e negar sensibilidades; suprimir as defesas construídas ao redor dos sentimentos ${ }^{52}$; expor e legitimamente compartilhar sentimentos dolorosos; compartilhar com co-profissionais algumas das frustrações, desapontamentos e raiva experimentados ao lidar com pacientes criticamente enfermos ou moribundos e dar voz às próprias preocupações sobre a vida, o morrer e a morte ${ }^{51}$.

\section{CONCLUSÃO}

A pedagogia das Humanidades médicas tem-se baseado numa noção tyleriana de currículo (visto como uma articulação de objetivos, instrução e avaliação) e, no que tange aos objetivos, na taxonomia de objetivos educacionais clássica, com seus domínios cognitivo, afetivo e psicomotor. Entretanto, muitos objetivos referentes aos traços humanísticos que se deseja facilitar ficam pouco mapeados, pois são de difícil classificação nesta taxonomia clássica. Deve-se, então, buscar outros sistemas conceituais como referenciais teóricos mais adequados às Humanidades.

Entre esses sistemas conceituais, a literatura oferece os Campos de Significado de Phenix e as Competências de Morse et al., mas sempre como sistemas únicos, substituindo a taxonomia de objetivos educacionais clássica, mas não necessariamente somando-se a ela. É possível que se possa usar as taxonomias de maneira aditiva, procurando objetivos humanísticos em cada uma das categorias e subcategorias das diversas taxonomias. Assim, aumenta-se a abrangência do corpo de objetivos educacionais de natureza humanística.

Havendo objetivos enunciados nas diversas categorias das diferentes taxonomias, a concepção tyleriana de currículo permite dar um passo à frente e organizar as atividades de ensino/aprendizagem e de avaliação inerentes a um programa educacional que almeje, de maneira realista e eficiente, desenvolver traços humanísticos nos alunos.

\section{REFERÊNCIAS}

1. Tapajós R. Os objetivos na educação médica. In: Marcondes E, Gonçalves EL, editores. Educação médica. São Paulo: Sarvier; 1998. p.69-94.

2. Tapajós R. HIV/AIDS in the visual arts: applying discipline-based art education (DBAE) to medical humanities. Med Educ. 2003;37:563-570.

3. Infante MS. A rational for general education in nursing curricula. J Nurs Educ. 1975;14(3):27-38

4. Mohr WK. Integrating aesthetics into nursing: literature as a suggested modality. Arch Psychiatr Nurs. 1995;9(6):365-72.

5. Lafferty PM. Balancing the curriculum: promoting aesthetic knowledge in nursing. Nurse Educ Today. 1997;17(4):281286.

6. Morse W, Bottoms MS, Wastlick LA. Liberal and professional undergraduate nursing education: maintaining the connections. J Prof Nurs. 1992;8(5):282-288. 
7. Troncon LEA, Cianflone ARL, Martin CCS, Alessi NP, Bava MCGC, Meneghelli UG. Conteúdos humanísticos na formação geral do médico. In: Marcondes E, Gonçalves EL, editores. Educação médica. São Paulo: Sarvier; 1998. p.99114.

8. Phenix PH. Realms of meaning. New York: McGraw-Hill Book Co.; 1964.

9. Davies IK. Objectives in curriculum design. London: McGraw-Hill Book Co.; 1976.

10. Phenix PH. Education and the common good: a moral philosophy of the curriculum. New York: Harper and Brothers; 1961.

11. Ratanakul P. The role of humanities in medical education. J Med Assoc Thai. 1977;60(7):326-329.

12. Parkin CJ. Humanities for the health professional. AD Nurse. 1987;2(6):30-31.

13. Barnard D. Making a place for the humanities in residency education. Acad Med. 1994;69(8):628-630.

14. Dunea G. A plea for a continuing liberal education in medicine. Chic Med Sch Q. 1966;26(3):160-161.

15. Shelton W. The role of humanities in medical education. J Ark Med Soc. 1982;79(4):125-129.

16. Bruderle ER, Valiga TM. Integrating the arts and humanities into nursing education. NLN Publ. 1994;June(14):117144.

17. Bates DG. Humanism in undergraduate medical school. CMAJ. 1971;105(3):258-261.

18. Reynolds RC, Carson RA. The place of humanities in medical education [editorial]. J Med Educ. 1976;51(2):142-143.

19. McElhinney TK. Placing the humanities perspective in the health profession curriculum. J Allied Health. 1983;12(3):221-228.

20. Engelhardt HT. Humanism and the profession(al). J Dent Educ. 1985;49(4):202-206.

21. Bench RJ. Health science, natural science and clinical knowledge. J Med Philos. 1989;14(2):147-164.

22. Taylor RN. Medical humanities and clinical practice. J S C Med Assoc. 1995;91(12):500-502.

23. Levine ME. On the humanities in nursing. Can J Nurs Res. 1995;27(2):19-23.

24. Sweeney B. The place of the humanities in the education of a doctor. Br J Gen Pract. 1998;48(427):998-1102.

25. Kahn L. Humanities now? Mo Med. 1988;85(12):783-784.

26. Clouser KD. Humanities and the medical school: a sketched rationale and description. Br J Med Educ. 1971;5(3):226231.

27. Banks SA, Vastyan EA. Humanistic studies in medical education. J Med Educ. 1973;48(3):248-257.
28. Rancich AM. La enseñanza de las humanidades médicas. Quiron. 1985;16(1):6-68.

29. Kempner HL. Remarks on the announcement of the Harris L. Kempner professorship of humanities at the University of Texas Medical Branch. Tex Rep Biol Med. 1974;32(1):3133.

30. Nelson BW. Liberal arts and humanities in medicine [editorial]. Pharos Alpha Omega Alpha Honor Med Soc. 1981;44(4):36.

31. Almagro BR. Medical libraries, medicine and the humanities. Ariz Med. 1984;41(9):619-620.

32. Shumacher HB. Leo Eloesser and the humanities in medicine. Pharos Alpha Omega Alpha Honor Med Soc. 1984;47(2):27-33.

33. Parkin CJ. Humanities for the health professional. AD Nurse. 1987;2(6):30-31.

34. Neidle EA. Dentistry - Ethics - The humanities: a threeunit bridge. J Dent Educ. 1980;44(12):693-696.

35. Siegel M, Siegel C. The use of literature in professional nursing education. Nurs Forum. 1977;16(2):156-164.

36. Wald SS. David B. Kriser Foundation establishes university professorship in humanities. N Y State Dent J. 1978;44(7):293-295.

37. Pierce ER. The liberal arts connection. Am J Occup Ther.1984;38(4):237-244.

38. Laborde RP. Medical humanities in Louisiana medical education. J La State Med Soc. 1984;136(5):47-48.

39. Hook EW. The Jeremiah Metzger Lecture. Humanities in medicine: treatment of a deficiency disorder. Trans Am Clin Climatol Assoc. 1996;108:203-221.

40. Mosley P. Role of the humanities in the education of health professionals. Med Teach. 1989;11(1):99-101.

41. Malacrida R, Wullschleger R, Rapin CH. De la bioéthique aux "medical humanities" par l'éthique clinique. Rev Med Suisse Romande. 1998;118(12):1019-1021.

42. Banks SA. The newcomers: humanities and social sciences in medical education. Tex Rep Biol Med. 1974;32(1):19-30.

43. Gogel EL, Terry JS. Medicine as interpretation: the uses of literary metaphors and methods. J Med Philos. 1987,12(3):205-217.

44. Saunders JB. Spoken language and small groups: a student experience in graduate nursing education. J Adv Nurs. 1982;7:529-544.

45. Loftus LS, Whitehead F, Arnold WN. A humanities and medicine program for faculty. Acad Med. 1991;66(12):730.

46. Krathwohl DR, Bloom BS, Masia BB. Taxonomy of educational objectives: handbook II: affective domain. New York: McKay; 1964. 
47. Oglov L. The case for a liberal education. CMAJ. 1977; 116(12): 1406-8.

48. Moretto RA, Mansur OFC, Araújo J. Humanismo e tecnicismo na formação médica. Rev Bras Educ Med. 1998;22(1): 19-25.

49. Risse GB. The role of medical history in the education of the 'humanist' physician: a reevaluation. J Med Educ. 1975; 50(5):458-465.

50. Glick S. Humanistic medicine in a modern age. N Engl J Med. 1981;304(17):1036-1038.
51. Bertman SL, Marks SC. Humanities in medical education. Med Educ.1985;19(5):374-381.

\section{CONFLITOS DE INTERESSE}

Declarou não haver.

\section{ENDEREÇO PARA CORRESPONDÊNCIA}

Rua Jericó, 255, cj 54

E-mail: ritapajos@uol.com.br 